\title{
Transcallosal Septum Pellucidum Interforniceal Approach to Remove Craniopharynginoma in Children
}

\author{
Kong Jun ${ }^{1^{*}}$, Xin Jin ${ }^{1}$, Hu Jie ${ }^{2}$, Lin Yi Hai ${ }^{1}$ and Wu Ye ${ }^{1}$ \\ ${ }^{1}$ Department of Neurosurgery, Tongde Hospital of Zhejiang Province, Zhejiang, PR China \\ ${ }^{2}$ Department of Neurosurgery, Huashan Hospital, Affiliated to Fudan University, Shanghai, PR China
}

"Corresponding author: Kong Jun, Department of Neurosurgery, Tongde Hospital of Zhejiang Province, Zhejiang, PR China, Tel: (0571) 8997200; E-mail: kongjj@163.com

Rec date: Jun 16, 2016; Acc date: Nov 28, 2016; Pub date: Nov 30, 2016

Copyright: (c) 2016 Jun K, et al. This is an open-access article distributed under the terms of the Creative Commons Attribution License, which permits unrestricted use, distribution, and reproduction in any medium, provided the original author and source are credited.

\begin{abstract}
Objective: The objective is to study the approach of removal of craniopharynginoma.

Methods: We remove eight cases of craniopharynginoma in childhood which intrudes the third ventricle transcallosal septum pellucidum interforniceal approach from August, 2013 to December, 2015.

Results: MRI or Recheck CT of brain post-operation can identify the removal situations of the tumor.

Conclusion: Tanscallosal septum pellucidum interforniceal approach to remove craniopharynginoma is a very important method.
\end{abstract}

Keywords: Transcallosal septum pellucidum interforniceal approach; Craniopharynginoma removal

\section{Introduction}

Craniopharynginoma in childhood is one of the most common intracranial tumors in children, which accounts to about $16 \%$ of it. Craniopharynginoma is a congenital benign tumor resulted from vestigial epithelial cell in craniopharyngoma during embryonic stage. We study eight cases of craniopharynginoma removal in childhood which intrude the third ventricle transcallosal septum pellucidum interforniceal approach from August, 2013 to December, 2015. All the patients have better results. Now the reports are as mentioned below.

\section{Material and Methods}

\section{General material}

Female five cases; male three cases. Ages are from eight to seventeen years old, the average 13.1. Three cases complain mainly of polydipsia and polyuria; two cases complain mainly of hypopsia in progress; two cases complain mainly of headache; one case complains mainly of endocrine disturbance.

\section{Neuroradiology procedures}

All these patients were checked by CT and MRI. Tumors in five patients intrude into frontal of third ventricle through saddle area, and tumors in three patients lie in third ventricle. Tumors have complete cystis degeneration in four cases, complete kernel in two cases, partly degeneration in two cases. Tumors have calcification in six cases, no calcification in two cases. Four cases have difference cerebral edema.

\section{Operative methods}

Craniotomy: Making crook incision inside hairline either right side or left side based on developing direction of tumors. The posterior line of the skin flap should behind coronal suture for $1.5 \mathrm{~cm}$. The inside of this incision should be put on the mid-line. Finishing incision of fullthickness, the skin flap should be turn to frontal. Drill two holes should be on the coronal suture on posterior line, another two holes should be ahead for $4-5 \mathrm{~cm}$. Two holes should be separated from each other for 4 $\mathrm{cm}$, and making a ladder-shaped bone flap, exploring the edge of sagittal sinus inside. The edge of bone flap in mid-line should be on the right edge of sagittal sinus. It is no need passing the mid-line to explore sagittal sinus so as no injuring to sagittal sinus.

Separation between hemisphere: Dural should be cut of in curve and be turn to the mid-line. Microscope should be put anteversion for twenty degree. Separating longitudinal split perpendicularly toward the line connecting two ears. Bridging vein in front of coronal suture can be cut after coagulated. But the bigger vein should be maintained as soon as possible $[1,2]$.

Incision of callositas: To find pericallosal artery on double side paralleling firstly. The incision of callositas should be located between two pericallosal arteries which can be either seperated carefully when clinging to each other or be push together to one side to make lateral incision on vessel but to maintain mid-line. Choose wider vessel gaps to operate when the upper three branches of vessels met on, maybe there exists callositas middle artery. It should not to incise the callositas by force if there is only one pericallosal artery to supply medial surface of double hemispheres. The boundary should be on precentral sulcus on medial surface of right hemisphere. There is little effective on message transfer between two hemispheres after limited length wise incision of callositas [2]. 
Separation of septum pellucidum and incision of interfornices: Opening septum pellucidum on the right side or the left side,after incision of callositas, it is seen anatomic landmarks such as choroid plexus in lateral cerebral ventricle, vena thalamostriata and septal vein, following these to find Mronro's hole and fornix. Longitudinal dissociation of fornix with detacher along mid-line following Monro's hole, there are eighty percent patients who have septum pellucidum cavity [3], which can make it easy to reach interfornices. Incision of interfornices is the top of third ventricular, where tumors can be seen.

Tumor incision: Puncture and drainage slowly firstly if tumor is cyst. When tumor collapses, the wall of tumor should be raised and be separated from normal tissure surrounded. Incision inside tumor when it is kernel. When tumor collapses, the wall of tumor should be raised and be removed from peridium of tumor. It must not to detach tumor by force when it encroach on hypothalamus, especially calcific plaque. It should be remove slightly with detacher. Sometime it is no need to remove the tumor totally. Cotton should be used to block upper mouth of aqueduct when it can be seen so as to prevent bleeding flowing into fourth ventricle. Besides of preventing injury to the bigger vessels constituted by Willis circular, it should be careful to maintain the blood supply from mini canalization arteries. Large area ischemic of hypothalamus will occur in case of injury of mini canalization arteries of Willis circular. It is possible not to put drainage tube so as not to cause subcutaneous fluidify when preparing to close the brain. We regard, even if aqueduct is block, incision of callosities equals to opening of top of third ventricle. This approach is difficult to close, which can make it easy to communicating from CCF to cistern so as to avoid intracranial hypertension [4].

\section{Post-operative treatment}

It is complex of the patterns of water-electrolyte metabolism from post-operation patients. It is more easy to cause severe results in children because their abilities to adjust water-electrolyte metabolism by themselves is poor. It should be careful to use saline during operation and to check electrolyte every morning and night postoperation, as well as to record the quantity of going out and coming in. It is not use dehydrater and diuretic in principal. Adjust the saline quantity of coming in based on the change of electrolyte. Also use hypophysin and Minirin based on urine volume. It is regular to use antiepileptic post-operation, such as carbamazepine by oral and hormones such as Dexamethasone, prednisone and thyroxine, and other necessary symptom treatments.

\section{Results}

Rechecked CT or MRI of brain post-operation can identify the removal situations of the tumor. Assessment standards of the operation of totally resection of craniopharynginoma are following: Remove totally all the tumor which can be seen in naked eye by operator; CT scan done in short period post-operation shows tumor calcification disappear; MRI of brain post-operation shows no tumor persistence. Among these cases, there were four cases totally resection, three cases subtotal resection and one case partial resection. There were seven cases who suffered polydipsia and hyperdiuresis post-operation and six cases getting better after treatment. There were one patient suffering epilepsia gravior, one patient suffering memory disorder and one patient suffering hydrocephalus.

\section{Discussion and Conclusion}

It is very diffcult to remove the craniopharynginoma in third ventricle. There is dispute [5] on whether to remove tumor totally or not which combines with huge calcification and adhere tightly on inferior part of thalamus opticus. Rajan [6] claimed on subtotal resection of tumor or partial resection of tumor. But we regard tumors easy to recur and the frequency of complications from the second operation due to tumors recurance is higher than the first operation after partial resection of craniopharynginoma. So we calm to remove tumor thoroughly as soon as possible. In our group, there are five cases removed totally or subtotally, one cases partial removed due to the tumor adhered tightly on third ventricle. Premise of total resection of the craniopharynginoma is to explore enough of the tumor. So it is based on tumor location to choose the approach. Callositas - septum pellucidum - interforniceal approach was raised firstly by Apuzzo [7] in 1982. Winkler [2] thought there was less complication from this approach after he studied by anatomy and clinic. Siwanuwantn [8] thought the callositas-interforniceal approach was the best approach to the third ventricle. We think this approach has such features as following:

1. It is the nearest approach to reach the third ventricle.

2. No need to open fornix column on one side and anterior region of thalamus makes no injury to thalamus, vena thalamostriata and internal cerebral veins, as well as lessens occurrence of hemiplegia, coma, memory disorder and mutism post-operation.

3. Operation under euthyphoria can expand toward bilateralis and AP. And visual angles on left and right can extend from ten to fifteen degree. Explore the tumor utmost nearly without any limited. It make easy to see important structures on both sides clearly and no need dragging cerebral tissue.

4. It can explore the bigger tumor located in frontal, middle and posterior of the third ventricle and make tumor total resection or subtotal resection by adjusting patient's head position and angle of microscope.

5. No need to open cortex can lessen occurrence of brain injury and hemiplegia as well as epilepsia post-operation. In short, craniopharynginoma in children, as well as its treatment, is synthetic. Removal by operation and radiotherapy are basic methods. But it is the premise of assurance of successful operation that treatment and correction of water, electrolyte and endocrine disorder.

\section{References}

1. Woiciechowsky C, Vogel S, Lehmann R, Staudt J (1995) Transcallosal removal of lesions affecting the third ventricle: Anatomic and clinical study. Neurosurgery 36: 117-122.

2. Winkler PA, Ilmberger J, Krishnan KG, Reulen HJ (2000) Transcallosal interforniceal-transforaminal approach for removing leisions occupying the third ventricular space: clinical and neuropsy chologica results. Neurosurgery 46: 879-888.

3. Winkler PA, Weis S, Büttner A, Raabe A, Amiridze N, et al. (1997) The Transcallosal interforniceal approach to the third ventricle: anatomic and microsurgical aspects. Neurosurgery 40: 973-981.

4. Holtzman RN, Brust JC, Ainyette IG, Bowers PP, Tikofsky RS, et al. (2001) Acute ventricular hemorrhage in adults with hydrocephalus managed by corpus callosotomy and fenestration of the septum pellucidum. J Neurosurg 95: 111-115.

5. Pascual JM, González-Llanos F, Barrios L, Roda JM (2004) Intraventricular craniopharyngiomas: Topographical classification and 
Citation: Jun K, Jin X, Jie H, Hai LY, Ye W (2016) Transcallosal Septum Pellucidum Interforniceal Approach to Remove Craniopharynginoma in Children. J Neurol Disord 4: 319. doi:10.4172/2329-6895.1000319

Page 3 of 3

surgical approach selection based on an extensive overview. Acta Neurochir 146: 785-802.

6. Rajan B, Ashley S, Gorman C, Jose CC, Horwich A, et al. (1999) Cranio pharyngiom as a long-term results following limited surgery and radiotherapy. Radiother Oncol 26: 1-10.

7. Apuzzo ML, Chikovani OK, Gott PS, Teng EL, Zee CS, et al. (1982) Transcallosal approaches for lesions affecting the third ventricle: Surgical considerations and consequences. Neurosurgery 10: 547-554.
8. Siwanuwatn R, Deshmukh P, Feiz-Erfan I, Rekate HL, Zabramski JM, et al. (2005) Microsurgical anatomy of the transcallosal anterior interforniceal approach to the third ventricle. Neurosurgery 56: 390-396. 